\title{
La pesca de sardina, Sardinella aurita (Teleostei: Clupeidae) asociada con la variabilidad ambiental del ecosistema de surgencia costera de Nueva Esparta, Venezuela
}

\author{
Leo W. González ${ }^{1,2}$, Jorge Euán ${ }^{1}$, Nora Eslava $^{2} \&$ Jesús Suniaga $^{3}$ \\ 1 Centro de Investigación y de Estudios Avanzados del IPN, Unidad Mérida, A.P. 73 Cordemex. 97310 Mérida, Yucatán, \\ México; walter@mda.cinvestav.mx, euan@mda.cinvestav.mx \\ 2 Área de Biología y Recursos Pesqueros, Instituto de Investigaciones Científicas, Universidad de Oriente, Núcleo de \\ Nueva Esparta. A.P. 749, Porlamar, isla de Margarita, Venezuela; leonora@telcel.net.ve \\ 3 Departamento de Ciencias, Dirección de Estudios Básicos, Universidad de Oriente, Núcleo de Nueva Esparta. A.P. \\ 147, Porlamar, isla de Margarita, Venezuela; asovida@cantv.net
}

Recibido 28-V-2002. Corregido 11-III-2006. Aceptado 13-X-2006.

\begin{abstract}
Association of sardine fishery, Sardinella aurita (Teleostei: Clupeidae) and environmental variability of the coastal upwelling ecosystem of Nueva Esparta, Venezuela. The present research is an analysis of Spanish sardine fishing (Sardinella aurita) associated with some climatic and meteorologic parameters of the ecosystem from El Morro Nueva Esparta, Venezuela. The catch and environmental data from the area were taken in the period 1996-2000. Catch data as a function of wind speed, sea surface temperature, air temperature and rain were analyzed by means of simple lineal regression and multiple models. We found a positive correlation of catch with wind speed, and a negative correlation with sea surface temperature, air temperature, and rain. The multiple regression model with intercept had a poor fit, therefore, we made a model without intercept, which improve greatly the fit. A selection of the variables using the forward procedure verified that the independent variables "wind speed" and "air temperature" have a significant relation with catch $(\mathrm{p}<0.001)$ at real time. This method suggests that sea surface temperature and rain have little influence on the catch, and suggests a major availability of resources in the months with low air temperature and the highest wind speed (January-June). Rev. Biol. Trop. 55 (1): 279-286. Epub 2007 March. 31.
\end{abstract}

Key words: Sardinella aurita, environmental variables, sardine fishing, Venezuela.

En las costas de Venezuela se registra una temperatura promedio de 25 a $29^{\circ} \mathrm{C}$, con un flujo de corrientes predominante que se dirigen de este a oeste, el cual presenta una velocidad moderada de 2 nudos; sin embargo, existe un gradiente espacial en la intensidad de los fenómenos de surgencia y uno temporal y periódico asociado a los ciclos anuales de los vientos alisios (Ginés 1972). La asociación de la temperatura superficial con las lluvias refleja una relación no lineal de tipo potencial; por debajo de $26^{\circ} \mathrm{C}$ casi no hay lluvias, $28^{\circ} \mathrm{C}$ define un nivel crítico (como en otras regiones marítimas tropicales), a temperaturas de $27^{\circ} \mathrm{C}$ llueve ligeramente, mientras que a $29^{\circ} \mathrm{C}$ se alcanzan niveles torrenciales (Anónimo 1999).
Las principales zonas de surgencia en Venezuela están localizadas en la región nororiental (Estados Nueva Esparta y Sucre) y centro-occidental (Península de Paraguaná y Golfo de Venezuela), en las cuales además existe un aporte importante de materia orgánica e inorgánica proveniente de aguas continentales. Estos dos factores (surgencia y aportes continentales) agregados a la existencia de una amplia plataforma continental explican la alta productividad biológica del medio marino venezolano.

El estudio de pelágicos menores, como sardinas y anchovetas, ha aumentado en las últimas décadas, debido a que representan un alto porcentaje de las capturas marinas en el ámbito mundial. Además, son afectadas en abundancia 
por el fenómeno de "El Niño", como lo señala la FAO (1999), quien considera a esta anomalía climática durante 1997-1998 como el segundo calentamiento más fuerte registrado en el siglo veinte en el Pacífico tropical y subtropical. Los impactos que ocasiona el evento ENOS (El Niño-Oscilación Sur) a las especies pelágicas, son los cambios de distribución y alteración de los niveles de abundancia y disponibilidad a los artes de pesca en las áreas tradicionalmente explotadas (Anónimo 1999). Es posible que la variabilidad del reclutamiento debido a los factores ambientales sea aún mayor, que la relacionada a cambios en la abundancia de la población de progenitores (Csirke 1980, García 1983). Por lo tanto, solamente el seguimiento de la evolución de los parámetros oceanográficos y atmosféricos, darán la pauta sobre el posible impacto del evento (De Anda et al. 1994).

La pesquería de la sardina tiene particular relevancia en la socio-economía del Estado Nueva Esparta por el número de empleos en las diferentes fases de captura, procesamiento y comercialización (González y Eslava 2000). Durante 1990-2000 la distribución porcentual de la captura de sardina entre los Estados Nueva Esparta y Sucre fue de $44 \%$ y $56 \%$, respectivamente (Guzmán 2001, com. pers.). Según las estadísticas oficiales del Instituto Nacional de Pesca y Acuicultura (INAPESCA) del Ministerio de Agricultura y Tierras, la pesca de sardina en el 2000 fue de 106750 ton, aportando Nueva Esparta 46480 ton (43.54\%), de los cuales 21635 ton correspondió al caladero El Morro ubicado al sureste de la isla de Margarita del Estado Nueva Esparta.

A fin de contribuir al conocimiento del comportamiento de la sardina de esta zona del Caribe sur oriental asociado al ecosistema de surgencia costera, se planteó la hipótesis de la posible relación de la captura con algunos factores climáticos y meteorológicos, basados en que la especie, presenta características, como la agregación en cardúmenes de fácil detección que los hace particularmente vulnerable al arte de pesca; además, presentan gran variabilidad natural del reclutamiento fuertemente dependiente de las condiciones ambientales, lo que origina incertidumbre en sus niveles de abundancia en el tiempo (De Anda et al. 1994).

\section{MATERIALES Y MÉTODOS}

Se analizaron datos mensuales de captura del caladero El Morro (Fig. 1), y registros de velocidad del viento, temperatura del aire y precipitación a tiempo real, desde enero de 1996 hasta diciembre de 2000. Los datos de pesca y variables ambientales fueron suministrados por la Asociación de Pescadores Artesanales de El Morro (ASOPESCAMO) y el Servicio de Meteorología de la Fuerza Aérea Venezolana (SMFAV) de la Estación de Porlamar, respectivamente. Los valores de la temperatura superficial del mar, se tomaron de

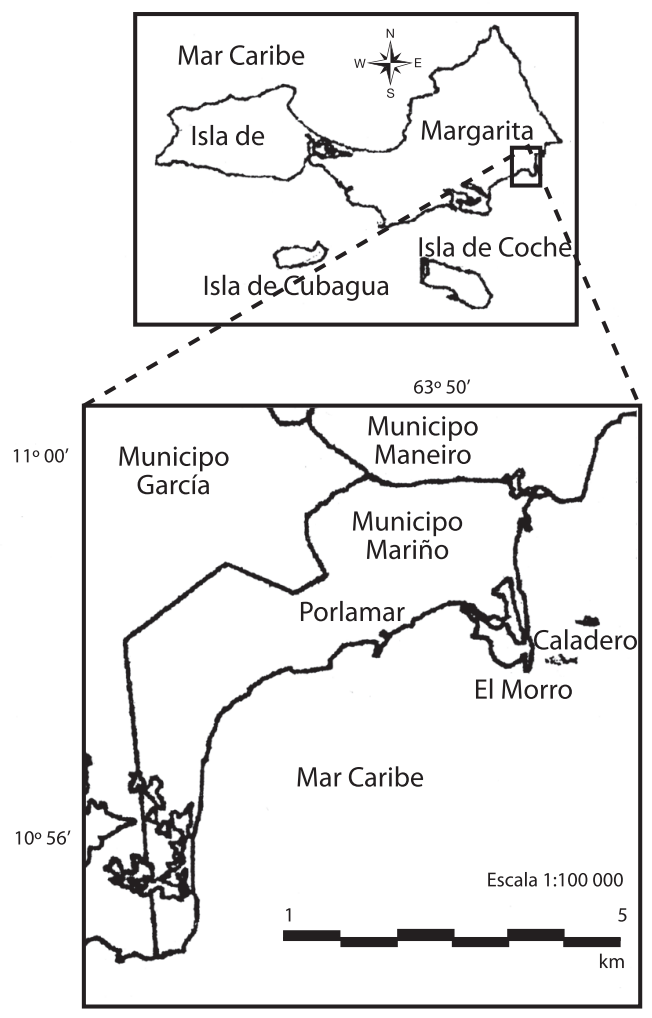

Fig. 1. Ubicación del área de pesca El Morro en el Estado Nueva Esparta, Venezuela.

Fig. 1. Location of the El Morro fishing area, Nueva Esparta State, Venezuela. 
la fuente del IGOSS (Integrated Global Ocean Services System) a través de la JCOMM (Joint Technical Commission for Oceanography and Marine Meteorology).

Con el propósito de describir las posibles relaciones que puedan existir entre la captura (C) y la velocidad del viento (V), temperatura superficial del mar (TSM), temperatura del aire $(\mathrm{T})$ y precipitación $(\mathrm{P})$; se propusieron los modelos de regresión lineal simple y de regresión lineal múltiple. El modelo de regresión múltiple fue evaluado a través del análisis de varianza. Se determinaron modelos de regresión sin intercepto y se validaron mediante selección de variables utilizando el método de regresión Forward. El software usado fue el Statgrafics Plus versión 2.1 y el SAS (Statiscal Analysis System) versión 7.0.

\section{RESULTADOS}

Captura y variables ambientales: los datos de producción anual de sardina en El Morro en el período 1996-2000 presentaron fluctuaciones temporales (Cuadro 1). En 1996 se alcanzó la captura más alta con 72886 ton, en 1997 disminuyó a 22060 ton hasta alcanzar en 1998 su valor más bajo con 18685 ton, posteriormente en 1999 se recuperó en 46800 ton, para luego caer en el año 2000 con 21635 ton, valor parecido al de 1997; también se observó que los mayores volúmenes de captura mensual ocurrieron en el primer semestre del año.

Las series mensuales promedio de la captura de sardina (C), velocidad del viento (V), temperatura superficial del mar (TSM), temperatura del aire $(\mathrm{T})$ y precipitación $(\mathrm{P})$ durante el

CUADRO 1

Producción mensual y anual (ton) de Sardinella aurita del área de pesca El Morro entre enero de 1996 $y$ diciembre de 2000

TABLE 1

Monthly and annual production (ton) of Sardinella aurita of the El Morro fishing area between January 1996 and December 2000

$\begin{array}{cccccccc}\text { Mes / Año } & 1996 & 1997 & 1998 & 1999 & 2000 & \text { Media } & \text { DE }( \pm) \\ \text { E } & 6600 & 335 & 210 & 250 & 0 & 1479 & 2865.38 \\ \text { F } & 6732 & 3227 & 4500 & 5400 & 2800 & 4532 & 1604.47 \\ \text { M } & 13662 & 6526 & 2900 & 6900 & 4000 & 6798 & 4189.93 \\ \text { A } & 24310 & 4822 & 3200 & 9000 & 2000 & 8666 & 9136.95 \\ \text { M } & 11374 & 1040 & 3250 & 9200 & 2500 & 5473 & 4531.67 \\ \text { J } & 4752 & 2330 & 3015 & 7500 & 3300 & 4179 & 2055.71 \\ \text { J } & 2376 & 990 & 120 & 5200 & 15 & 1740 & 2152.82 \\ \text { A } & 0 & 670 & 750 & 2500 & 5 & 785 & 1022.33 \\ \text { S } & 924 & 750 & 150 & 550 & 2300 & 935 & 815.79 \\ \text { O } & 1848 & 840 & 160 & 300 & 4500 & 1530 & 1788.00 \\ \text { N } & 308 & 530 & 80 & 0 & 140 & 212 & 210.89 \\ \text { D } & 0 & 0 & 350 & 0 & 75 & 85 & 151.66 \\ \text { Total } & 72886 & 22060 & 18685 & 46800 & 21635 & & \\ \text { Media } & 6074 & 1833 & 1557 & 3900 & 1803 & & \\ \text { DE ( } \pm \text { ( } & 7297.37 & 2029.35 & 1658.49 & 3691.33 & 1693.30 & & \end{array}$

Fuente: ASOPESCAMO 
período 1996-2000 se muestran en el Cuadro 2. Se pudo evidenciar en la Fig. 2 que la pesca mejora a medida que la velocidad del viento aumenta (enero-junio), otorgándole a esta actividad una característica estacional; mientras que con la temperatura (superficial del mar y aire) y la precipitación no ocurre lo mismo, con excepción del mes de abril que presentó el valor más alto de captura (8 666 ton) y el más bajo de precipitación $(5.8 \mathrm{~mm})$.

Análisis estadístico: el grado de asociación mensual de la captura de sardina con cuatro variables ambientales, mediante el modelo de regresión lineal simple, se indican en las siguientes ecuaciones:

Captura vs. Viento:

$$
\begin{gathered}
\hat{\mathrm{C}}=-8135.039+1898.493 * \mathrm{~V} \\
(\mathrm{r}=0.53 ; \mathrm{n}=60 ; \mathrm{F}=19.42 ; \mathrm{p}<.0001)
\end{gathered}
$$

Captura vs. TSM:

$$
\widehat{\mathrm{C}}=45569.532-1039.2 * \mathrm{TSM}
$$

$$
(\mathrm{r}=-0.46 ; \mathrm{n}=60 ; \mathrm{F}=13.51 ; \mathrm{p}<.0006)
$$

Captura vs. T aire:

$$
\begin{gathered}
\hat{C}=25245.013-815.616^{*} \mathrm{~T} \\
(\mathrm{r}=-0.23 ; \mathrm{n}=60 ; \mathrm{F}=2.85 ; \mathrm{p}<.0979)
\end{gathered}
$$

Captura vs. Precipitación:

$$
\begin{gathered}
\hat{C}=4126.392-25.453 * P \\
(r=-0.32 ; n=60 ; F=5.75 ; \mathrm{p}<.0203)
\end{gathered}
$$

De las cuatro regresiones lineales estimadas, se puede observar que la correlación más alta y positiva fue la ecuación de la captura con la velocidad del viento. Por otro lado, la ecuación lineal que describió la relación entre la temperatura superficial del mar (TSM) y la temperatura del aire $(\mathrm{T})$ fue la siguiente:

$$
\mathrm{TSM}=5.9779+0.7416 * \mathrm{~T} ; \mathrm{r}=0.73 \mathrm{R}^{2}=52 \%
$$

Esto demuestra que algunas de las variables independientes, e.g. la temperatura superficial del mar y la temperatura del aire están correlacionadas entre si por lo tanto, se hizo un

\section{CUADRO 2}

Promedios mensuales y desviación estándar (DE) de captura (ton) de Sardinella aurita, velocidad del viento $\left(\mathrm{ms}^{-1}\right)$, temperatura superficial del mar $\left({ }^{\circ} \mathrm{C}\right)$, temperatura del aire $\left({ }^{\circ} \mathrm{C}\right)$ y precipitación $(\mathrm{mm})$ del caladero de pesca El Morro, entre enero de 1996 y diciembre de 2000

TABLE 2

Monthly averages and standard deviation (SD) of catch (ton) of Sardinella aurita, wind speed $\left(\mathrm{ms}^{-1}\right)$, sea surface temperature $\left({ }^{\circ} \mathrm{C}\right)$, air temperature $\left({ }^{\circ} \mathrm{C}\right)$ and rain $(\mathrm{mm})$ of the El Morro fishing area, between January 1996 and December 2000

$\begin{array}{ccccccccccc}\text { Mes } & \begin{array}{c}\text { Captura } \\ \text { (ton) }\end{array} & \begin{array}{c}\text { DE } \\ ( \pm)\end{array} & \begin{array}{c}\text { Viento } \\ \left(\mathrm{ms}^{-1}\right)\end{array} & \begin{array}{c}\mathrm{DE} \\ ( \pm)\end{array} & \begin{array}{c}\mathrm{TSM} \\ \left({ }^{\circ} \mathrm{C}\right)\end{array} & \begin{array}{c}\mathrm{DE} \\ ( \pm)\end{array} & \begin{array}{c}\mathrm{T} \text { aire } \\ \left({ }^{\circ} \mathrm{C}\right)\end{array} & \begin{array}{c}\mathrm{DE} \\ ( \pm)\end{array} & \begin{array}{c}\mathrm{P} \\ (\mathrm{mm})\end{array} & \begin{array}{c}\mathrm{DE} \\ ( \pm)\end{array} \\ \mathrm{E} & 1479 & 2865.38 & 5.76 & 1.11 & 25.3 & 0.24 & 25.68 & 0.84 & 59.8 & 49.52 \\ \mathrm{~F} & 4532 & 1604.47 & 6.16 & 0.75 & 24.8 & 0.40 & 25.88 & 0.93 & 33.6 & 27.33 \\ \mathrm{M} & 6798 & 4189.93 & 6.96 & 1.19 & 24.6 & 0.37 & 26.08 & 0.93 & 17.4 & 15.14 \\ \mathrm{~A} & 8666 & 9136.95 & 7.38 & 0.33 & 25.1 & 0.20 & 27.02 & 0.88 & 5.8 & 6.65 \\ \mathrm{M} & 5473 & 4531.67 & 7.28 & 0.58 & 25.9 & 0.73 & 27.72 & 0.56 & 11.2 & 13.81 \\ \mathrm{~J} & 4179 & 2055.71 & 6.38 & 0.37 & 26.1 & 0.49 & 27.34 & 0.59 & 52.6 & 28.89 \\ \mathrm{~J} & 1740 & 2152.82 & 5.38 & 0.63 & 26.5 & 0.45 & 27.60 & 0.55 & 63.2 & 50.44 \\ \mathrm{~A} & 785 & 1022.33 & 4.88 & 0.79 & 27.2 & 0.68 & 28.22 & 0.41 & 34.6 & 28.86 \\ \mathrm{~S} & 935 & 815.79 & 5.00 & 0.44 & 27.7 & 0.40 & 28.68 & 0.29 & 36.0 & 32.65 \\ \mathrm{O} & 1530 & 1788.00 & 5.00 & 0.59 & 27.6 & 0.37 & 28.32 & 0.41 & 49.8 & 18.67 \\ \mathrm{~N} & 212 & 210.89 & 5.00 & 1.01 & 27.1 & 0.20 & 27.66 & 0.49 & 54.6 & 56.80 \\ \mathrm{D} & 85 & 151.66 & 5.42 & 0.92 & 26.2 & 0.60 & 26.58 & 0.43 & 96.2 & 120.32\end{array}$



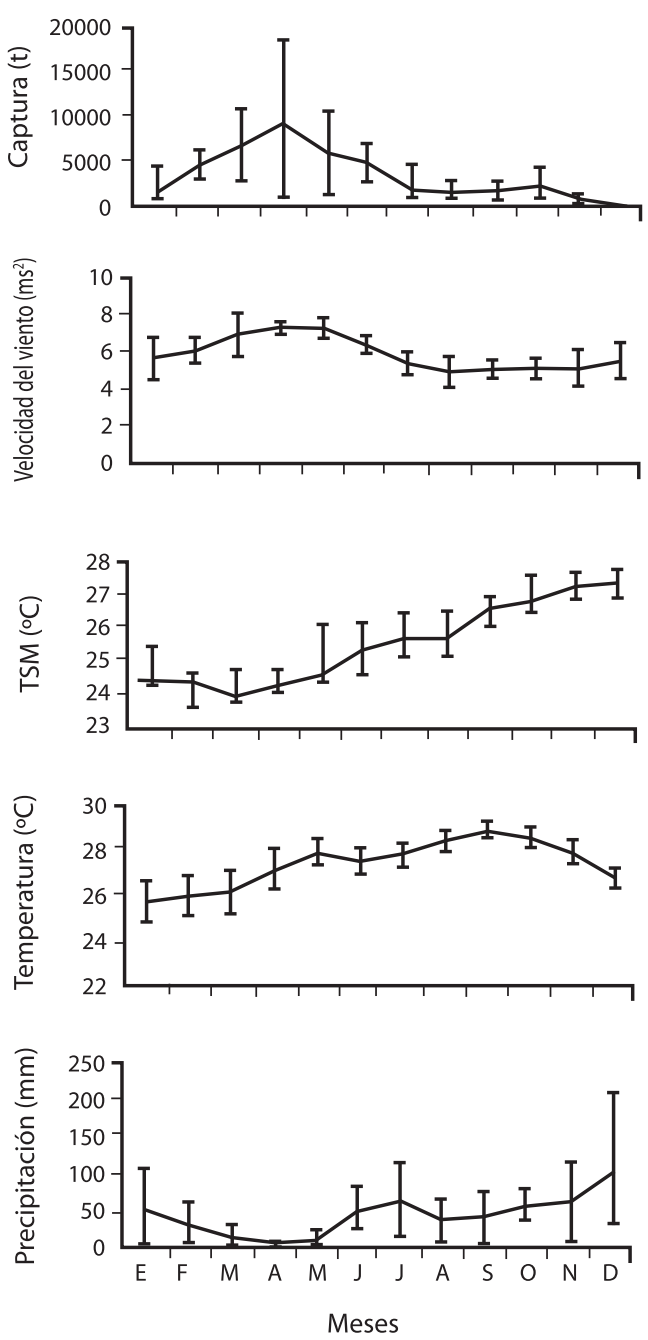

Fig. 2. Variación estacional de la captura de Sardinella aurita, velocidad del viento, temperatura superficial del mar, temperatura del aire y precipitación en El Morro, durante 1996-2000.

Fig. 2. Seasonal variation of the catch of Sardinella aurita, wind speed, sea surface temperature, air temperature and rain in El Morro, during 1996-2000.

enfoque multivariante del problema a través del análisis de regresión múltiple.

El modelo de regresión múltiple que definió la relación entre la captura y las cuatro variables independientes fue:

$$
\begin{gathered}
\hat{\mathrm{C}}=9792+1553.07 * \mathrm{~V}-229.65 * \mathrm{TSM}-354.72 \\
* \mathrm{~T}-5.23 * \mathrm{P}
\end{gathered}
$$

Como se puede apreciar en los parámetros estimados del Cuadro 3, la variable independiente $\mathrm{V}\left(\mathrm{t}_{\mathrm{s}}=2.151 ; \mathrm{p}<.05\right)$ influye significativamente sobre la variable dependiente $\mathrm{C}$; mientras que las otras tres variables TSM $\left(\mathrm{t}_{\mathrm{s}}=\right.$ $-0.227 ; \mathrm{p}>.05), \mathrm{T}\left(\mathrm{t}_{\mathrm{s}}=-0.447 ; \mathrm{p}>.05\right)$ y $\mathrm{P}\left(\mathrm{t}_{\mathrm{s}}\right.$ $=-0.438 ; p>.05)$, aparentemente no influyen de manera significativa en el modelo por tener valores probabilísticos mayores de .05. En el análisis de varianza para la variable dependiente $C$, se observa que el modelo lineal es sustentable con un nivel de significancia inferior a $.0008(\mathrm{~F}=5.535 ; \mathrm{p}<.001)$ lo que demuestra que la regresión es altamente significativa.

En el Cuadro 3 se observa que la regresión sin intercepto es altamente significativa $(\mathrm{F}=$ $16.060 ; \mathrm{p} \leq .0001)$, pues el valor de variación del modelo $\left(\mathrm{R}^{2}\right)$ es de $53.43 \%$. Los parámetros estimados de las variables independientes, para la regresión sin intercepto y la prueba $t_{\mathrm{s}}$ establecieron que los coeficientes de TSM $\left(\mathrm{t}_{\mathrm{s}}=0.174\right.$; $\mathrm{p}>.05), \mathrm{T}\left(\mathrm{t}_{\mathrm{s}}=-0.523 ; \mathrm{p}>.05\right)$ y $\mathrm{P}\left(\mathrm{t}_{\mathrm{s}}=-0.349\right.$; $\mathrm{p}>.05)$ tienen probabilidades superiores al límite establecido, lo que indica que estas variables no son significativas; mientras que con la variable $\mathrm{V}\left(\mathrm{t}_{\mathrm{s}}=3.759 ; \mathrm{p}<.05\right)$ ocurre lo contrario. Estos resultados se verificaron con la técnica de selección de variable utilizando el método de regresión de Forward. Con este método las variables $\mathrm{V}$ y $\mathrm{T}$ cumplieron con el criterio de entrada, quedando establecida la ecuación de la siguiente manera:

$$
\widehat{\mathrm{C}}=1872.37 * \mathrm{~V}-293.38 * \mathrm{~T}
$$

Por lo tanto, los análisis realizados en esta investigación sugieren que las variables $\mathrm{V}(\mathrm{F}=$ $23.85 ; \mathrm{p}<.0001)$ y $\mathrm{T}(\mathrm{F}=12.12 ; \mathrm{p} \leq .0010)$ del modelo de regresión sin intercepto son significativas para la variable $\mathrm{C}$.

\section{DISCUSIÓN}

Los resultados demuestran que el patrón de variación de la captura, responde a la variabilidad ambiental y de ello están concientes los pescadores, que aún siendo la pesca de sardina 
CUADRO 3

Análisis de la varianza para la regresión múltiple de la captura sin intercepto

TABLE 3

Analysis of the variance for the multiple regression model of catch without intercept

\begin{tabular}{|c|c|c|c|c|c|}
\hline Fuente & Gl & Suma de cuadrados & Media cuadrática & Valor de F & Prob. $>$ F \\
\hline Modelo & 4 & 842342968.96 & 210585742.24 & 16.060 & 0.0001 \\
\hline Error & 56 & 734277513.04 & 13112098.447 & & \\
\hline \multirow[t]{4}{*}{ Total } & 60 & 1576620482.0 & & & \\
\hline & & Root MSE & 3621.06317 & $\mathrm{R}^{2}$ & 0.5343 \\
\hline & & Dep. media & 3034.43333 & Adj. $R^{2}$ & 0.5010 \\
\hline & & C.V. & 119.33243 & & \\
\hline Variable & Gl & Parámetro estimado & Error estándar & $\begin{array}{c}\mathrm{t} \text { de } \mathrm{Ho}: \\
\text { parámetro }=0\end{array}$ & Prob. $>\mathrm{t}$ \\
\hline V & 1 & 1844.737268 & 490.70495804 & 3.759 & 0.0004 \\
\hline TSM & 1 & 132.994944 & 766.09539981 & 0.174 & 0.8628 \\
\hline $\mathrm{T}$ aire & 1 & -408.773308 & 781.9545931 & -0.523 & 0.6032 \\
\hline $\mathrm{P}$ & 1 & -4.085478 & 11.69147726 & -0.349 & 0.7281 \\
\hline
\end{tabular}

de acceso abierto y con pocas medidas de regulación, se asignan turnos de pesca según la abundancia del recurso (González et al. 2005). Así queda demostrada la hipótesis inicial, que suponía una mayor captura en el primer semestre del año asociada a la época de surgencia intensa como resultado de la entrada de nutrientes, debido a un posible efecto potencial de la velocidad del viento que caracteriza al ecosistema costero de esta zona del Caribe sur oriental (Herrera y Febres 1975, Okuda 1978).

Las correlaciones de la captura-viento, temperatura superficial del mar-temperatura del aire, y las probabilidades de las variables regresoras de la regresión múltiple explican la mayor disponibilidad y/o accesibilidad del recurso que corresponde a los meses de enero a junio cuando ocurre la mayor intensidad de vientos y las temperaturas bajas. Estas estimaciones coinciden, en cierta manera, con las obtenidas por Mendoza et al. (1998), al encontrar una alta correspondencia entre la presencia de la sardina y los fenómenos de surgencia en el oriente venezolano. Asimismo, Cárdenas y
Achury (2000) consideran que la presencia de altas densidades de sardina, en determinado lugar, es consecuencia de múltiples variables ambientales, bióticas y abióticas que actúan simultáneamente para conformar un espacio propicio, siendo la temperatura el parámetro indicador de los focos de surgencia y que además, condiciona en alto grado las características del ambiente marino. Sin embargo, los resultados difieren con los obtenidos por Trujillo (1980), quien determinó una correlación negativa entre las capturas mensuales de sardina y la intensidad del viento en la zona de pesca.

Por otro lado, Frèon et al. (1997), señalan que es difícil determinar con precisión la influencia de la variabilidad ambiental anual en la biología de la sardina; esto posiblemente se deba a que la hidrografía de la región nororiental de Venezuela es muy peculiar, pues la surgencia costera y la influencia del río Orinoco se alternan en sus máximos estacionales, induciendo una alta productividad sostenida todo el año, esto al menos sobre la plataforma continental. 
El mismo criterio científico es compartido por R. Varela (2000, com. pers.), al indicar que las corrientes provenientes del Atlántico actúan marcadamente en la dispersión de las aguas de surgencia y las mezcladas con aportes fluviales que dan como resultado un desplazamiento hacia el nordeste en la forma que toman las áreas de mayor concentración de clorofila. El efecto combinado de surgencia costera inducido por el viento en la temporada seca y la descarga del río Orinoco en la época de lluvias genera niveles relativamente altos de producción primaria, que son altamente variables en espacio y tiempo, que van desde $3 \mathrm{gCm}^{2} \mathrm{~d}^{-1}$ en áreas de surgencia costera hasta $0.2 \mathrm{gCm}^{2} \mathrm{~d}^{-1} \mathrm{en}$ áreas más alejadas de las mismas.

Cabe deducir que las anomalías meteorológicas delimitan la pesca como consecuencia de la teleconexión de alta frecuencia de los eventos ENOS y La Niña desfasados entre el Caribe y el Pacífico Tropical. En tal sentido, es probable la relación de estos eventos en la región nororiental de Venezuela, por lo menos en el lapso 1997-1999 que fue precedido por un período sumamente frío, cuando los cambios ambientales en las costas de Sudamérica empezaron a observarse desde principios de 1997 (Anónimo 1999). Durante las anomalías climáticas del fenómeno de El Niño 19821983 y 1997-1998 el comportamiento de las precipitaciones anuales ocurridas en Venezuela fue de 317-252 $\mathrm{mm}$ y $383-575 \mathrm{~mm}$, respectivamente. Esta forma irregular y desfasada en espacio y tiempo, ocasionan lluvias en formas localizadas e intensas, e.g. en enero-marzo de 1998 mientras se presentaba sequías en el norte de Sudamérica, en Ecuador y norte de Perú se observaban lluvias, y se reducían los huracanes en el Caribe (Anónimo 1999), debido a que en esta región durante las fases cálidas del evento ENOS se produce una intensificación de los vientos alisios del noreste, con el consiguiente desplazamiento hacia el sur de la zona de convergencia intertropical, mientras que durante las fases frías, estos vientos se debilitan. Muchas anomalías negativas de precipitación en el norte de Sudamérica están asociadas a fases cálidas del ciclo ENOS y las anomalías positivas a períodos fríos o eventos de La Niña (Hastenrath 1984).

Se concluye que la captura de sardina en áreas muy costeras está asociada con la intensidad de vientos y con la temperatura del aire; de tal manera que su disponibilidad y/o accesibilidad esta sujeta a la variabilidad natural, lo que amerita información de índole periódica, indispensable en la evaluación y toma de decisiones en la administración de su pesquería. Más aún, cuando existen razones para pensar que la introducción de un nuevo arte de pesca, como el cerco o tren de argolla sardinero, y la interferencia de las redes de enmalle derivante que cierran la ruta de la sardina hacia los caladeros cercanos a la costa, podrían originar a futuro alteración de la estabilidad que mantienen armónicamente la pesca artesanal sardinera y la industria enlatadora nacional, así como el desequilibrio trófico del ecosistema costero.

\section{AGRADECIMIENTOS}

Este trabajo forma parte de la Tesis Doctoral de Leo Walter González Cabellos en CINVESTAV-IPN Unidad Mérida, la cual es realizada con el apoyo de la Universidad de Oriente. Se agradece al SMFAV por los datos climatológicos y meteorológicos de la Estación de Porlamar, al IGOSS/JCOMM por el acceso a los datos de temperatura superficial del mar del Estado Nueva Esparta y a la ASOPESCAMO por los datos de producción del caladero El Morro. A Eglée Gómez profesora de la ECAMUDO por la lectura crítica del manuscrito. A Héctor Mares del CINVESTAV por la elaboración de los dibujos.

\section{RESUMEN}

Se comparó la pesca de sardina (Sardinella aurita) con algunos parámetros climáticos y meteorológicos del ecosistema de surgencia costera de El Morro, Nueva Esparta, Venezuela. Para el período 1996-2000, se aplicaron los modelos de regresión lineal simple y múltiple a los datos de captura en función de la velocidad del viento, temperatura superficial del mar, temperatura del aire y pluviosidad. Hubo una correlación positiva de la captura 
con la velocidad del viento, y una negativa con temperatura superficial del mar, temperatura del aire y pluviosidad. El modelo de regresión múltiple con intercepto mostró un ajuste bajo, por tal razón se aplicó un modelo de regresión sin intercepto, el cual mejoró significativamente el ajuste. También se aplicó el método de selección de variables "hacia adelante", encontrándose que las variables independientes viento y temperatura del aire guardan relación significativa con la captura de sardina a tiempo real $(\mathrm{p}<0.001)$. Esto sugiere una mayor disponibilidad y/o accesibilidad del recurso en los meses con mayor intensidad de vientos y temperaturas bajas (enero-junio). Este método también sugiere que la temperatura superficial del mar y la pluviosidad tienen poca influencia en la captura de sardina.

Key words: Sardinella aurita, variables ambientales, pesca de sardina, Venezuela

\section{REFERENCIAS}

Anónimo. 1999. Forum "El fenómeno de El Niño 1997-1998: evolución, pronóstico y mitigación". Publicación especial, Instituto del Mar del Perú, Lima, Perú. 150 p.

Cárdenas, J. \& A. Achury. 2000. Acústica pesquera de los recursos marinos del nororiente de Venezuela: evaluación y seguimiento espacio-temporal del stock de sardina (Sardinella aurita Valenciennes, 1847). Mem. Fund. La Salle Cienc. Nat. 154: 39-54.

Csirke, J. 1980. Recruitment in the Peruvian anchovy and its dependence on the adult population. Rapp. P. -V Réun. CIEM. 177: 307-313.

De Anda, M.J.A., J.C. Seijo \& S. Martínez. 1994. Reclutamiento y variabilidad ambiental en la pesquería de sardina Monterrey (Sardinops sagax) del Golfo de California, México. Inv. Pesq. 38: 23 - 36.

FAO. 1999. El estado mundial de la pesca y la acuicultura 1998. Departamento de Pesca de la FAO, Roma, Italia. $112 \mathrm{p}$.

Frèon, P., M. El Khattabi, J. Mendoza \& R. Guzmán. 1997. Unexpected reproductive strategy of Sardinella aurita off the coast of Venezuela. Mar. Biol. 128: 363-372.

García, S. 1983. The stock-recruitment relationship en shrimps: reality or artifacts and misinterpretations? Oceanogr. Trop. 18: 25-48.

Ginés, Hno. (ed.). 1972. Carta pesquera de Venezuela. Fund. La Salle Cienc. Nat. Caracas, Venezuela. 319 p.

González, L.W. \& N. Eslava. 2000. Crecimiento y mortalidad natural de la sardina, Sardinella aurita (Teleostei: Clupeidae) del Estado Nueva Esparta. Rev. Biol. Mar. y Oceanogr. 35: 83-91.

González, L.W., S. Salas \& N. Eslava. 2005. Caracterización socio-económica de la pesquería artesanal de la sardina (Sardinella aurita) en el sureste de la isla de Margarita, Venezuela. Bol. Centro Invest. Biol. 39 (3): 197-216.

Herrera, L. \& G. Febres. 1975. Procesos de surgencia y renovación de aguas en la fosa de Cariaco, mar Caribe. Bol. Inst. Oceanogr. Venezuela 14: 31-44.

Hastenrath, S. 1984. Interannual variability and annual cycle: mechanisms of circulation and climate in the Tropical Atlantic sector. Mon. Wheather Rev. 112: 1097-1107.

Mendoza, J., P. Frèon, R. Guzmán \& R. Aparicio. 1998. Sardinella aurita population dynamics related to environmental parameters in the Southern Caribbean (Venezuela), p. 239-309. In M. Durand, P. Cury, R. Mendelssohn, C. Roy, A. Bakun \& D. Pauly (eds.). Global versus local changes in upwelling systems. Collection colloques et seminaries, ORSTOM, Paris, Francia.

Okuda, T. 1978. Características hidroquímicas de las aguas superficiales de la fosa de Cariaco y áreas adyacentes. FAO Fish. Rep. 200: 349-362.

Trujillo, H. 1980. Fluctuaciones de la velocidad y dirección de los vientos y su relación con las variaciones mensuales de la captura y producción potencial de sardina (Sardinella anchovia). Dirección General de Desarrollo Pesquero, Caracas, Venezuela. 26 p. 